\title{
Conf.940963--1
}

For the Proceedings of the XIlth intemational Seminar on High Energy Physics Problems , JINR, Dubna, RUSSIA

\section{PROTON-PROTON INTERACTIONS USING THE RHIC POLARIZED COLLIDER}

\author{
A. Yokosawa \\ High Energy Physics Division \\ Argonne National Laboratory, Argonne, Ilinois 60439
}

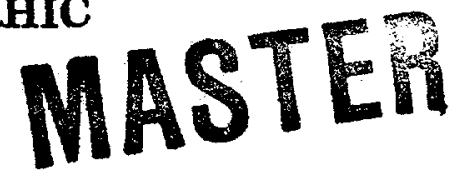

\begin{abstract}
The first polarized collider where we collide $250-\mathrm{GeV} / \mathrm{c}$ beams of $70 \%$ polarized protons at high luminosity is under construction. This will allow a determination of the nucleon spin-dependent structure functions over a large range in $\mathrm{x}$ and a collection of sufficient $\mathrm{W}$ and $\mathrm{Z}$ events to investigate extremely interesting spin-related phenomena.
\end{abstract}

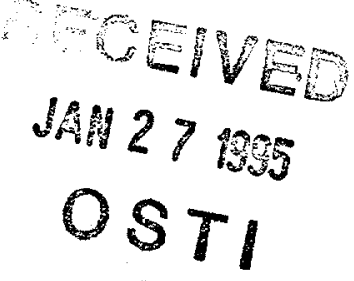

\section{Introduction}

We start out with discussing the status of the RHIC polarized collider and associated experiments.

In August 1991, a Partial Snake experiment at AGS was approved by the Brookhaven Program Advisory Committee (PAC). Then in August 1992 the Relativistic Heavy Ion Collider (RHIC) Spin Collaboration (RSC), STAR/Spin PHENIX/Spin proposals were submitted to the PAC. In October 1993, full approval of the proposals on spin physics using the RHIC polarized collider was granted by the Brookhaven National Laboratory PAC. In April this year, successful polarized proton acceleration in the AGS with a partial snake was achieved.

Proposed spin experiments at RHIC are to explore QCD in a new way and allow us to make the first direct measurement on one of the proton structure functions.

1. Test of a Partial Snake (E-880)

A partial Siberian snake was used to successfully accelerate polarized protons for the first time to $10.8-\mathrm{GeV}$ kinetic energy, without using harmonic orbit corrections.

There are two kinds of depolarizing resonances, imperfection and intrinsic. Imperfection resonances caused by rotated dipoles -- quadrupoles not centered on the beam. Previously a system using large numbers of orbit correction dipoles was used to minimize imperfection resonances. This system took weeks to tune, and was different every year due to the changing (by fractions of a millimeter) setting of the ring magnet positions. A partial snake should eliminate imperfection resonances. 
Intrinsic resonances are caused by horizontal fields in quadrupoles used for focusing. Pulsed quadrupoles were used to avoid intrinsic resonances.

A $9^{\circ}$ solenoidal spin rotator (partial Siberian snake) was used to successfully accelerate polarized protons for the first time to $10.8 \mathrm{-GeV}$ kinetic energy in the Brookhaven AGS without using harmonic orbit correctors. ${ }^{1}$

Figure 1 shows the Brookhaven hadron facility complex, which includes the AGS booster, the AGS, and RHIC. The solenoidal partial snake is installed in a 3-meter straight section in the AGS. The RHIC spin project will install two snakes per ring with four spin rotators per detector for achieving helicity-spin experiments.

\section{Polarized Proton Collisions at BNL}

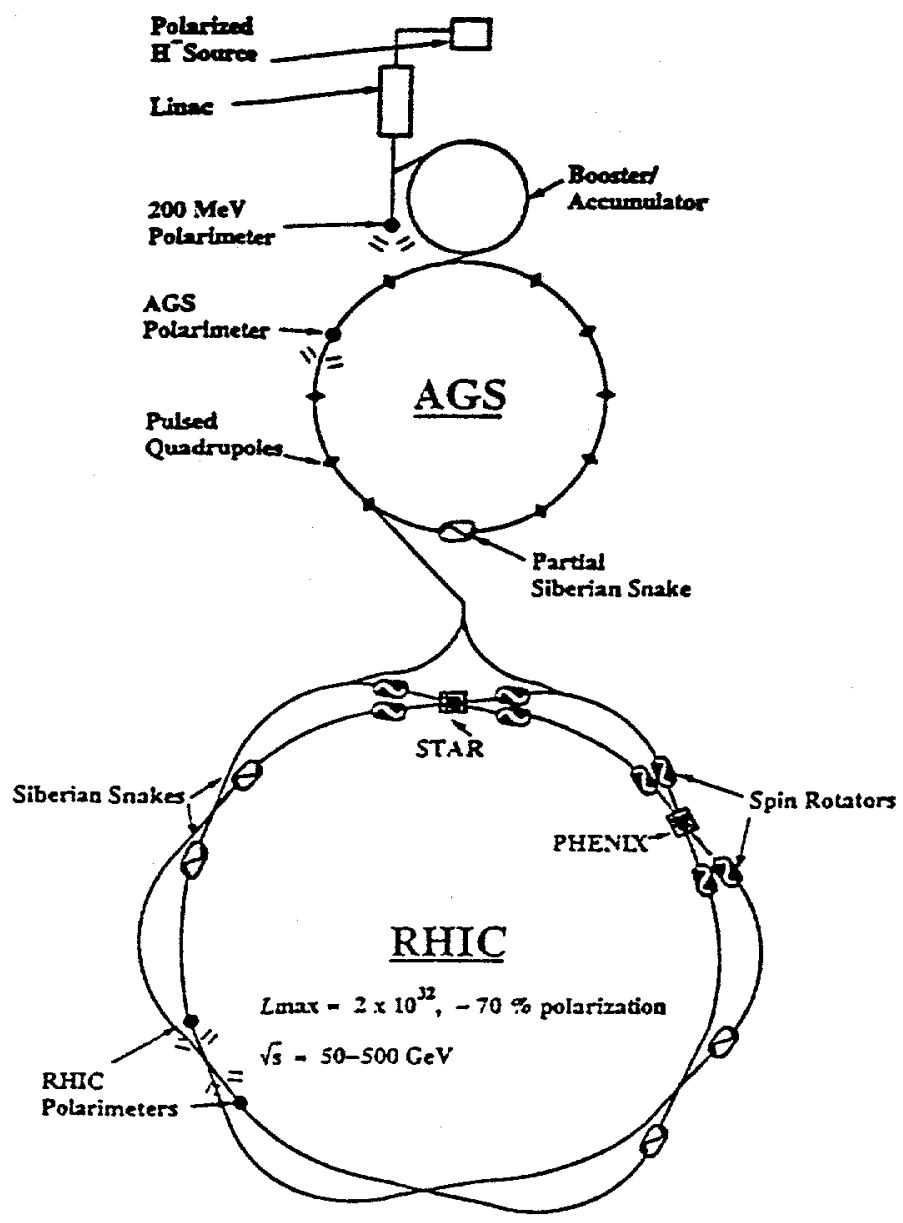

Figure 1 AGS booster, AGS, RHIC.

Figure 2 shows the measured value of the vertical polarization at $\mathrm{G} \gamma=\mathrm{n}+1 / 2$ up to $\mathrm{G} \gamma=22.5$ with and without snake. Note here that partial depolarization is due to intrinsic spin resonances at $\mathrm{G} \gamma=v \mathrm{y}, 24-\mathrm{vy}$, and $12+v \mathrm{y}$. Intrinsic resonances were not corrected in this test. 


\title{
DISCLAIMER
}

\begin{abstract}
Portions of this document may be illegible in electronic image products. Images are produced from the best available original document.
\end{abstract}




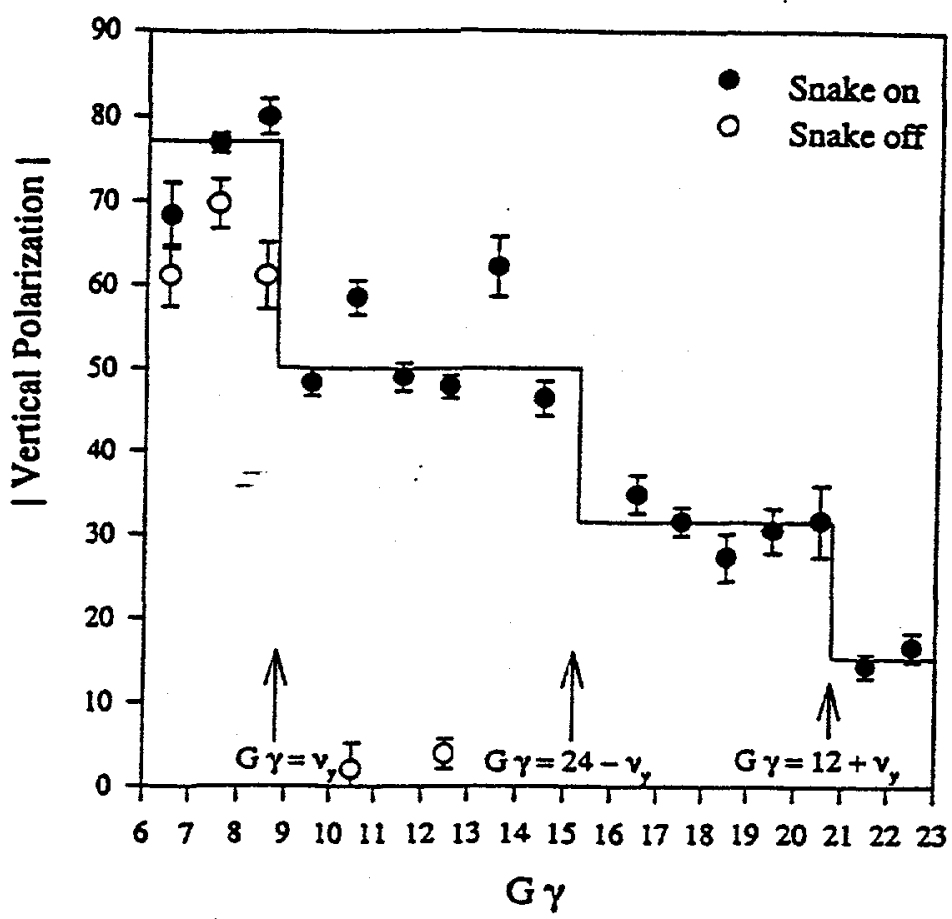

Figure 2 The effect of the partial snake.

\section{High Energy Spin Physics at RHIC}

We present physics issues and expected event rates for various reactions (the integrated luminosities used are shown in Appendix I).

The RHIC polarized collider allows us to explore QCD in a new way and opens challenging opportunities for QCD studies.

The proton spin consists of:

$$
1 / 2 \Sigma \Delta \mathrm{q}+\Delta \mathrm{G}+<\mathrm{I} \mathrm{z}>=1 / 2
$$

Our proposed measurements are to determine sea-quark polarization, $\Delta \overline{\mathrm{u}}(\mathrm{x})$ and $\Delta \overline{\mathrm{d}}(\mathrm{x})$, and gluon polarization $\Delta \mathrm{G}(\mathrm{x})$. We are also to determine one of the fundamental proton structure functions $\left(f_{1}, g_{1}, h_{1}\right)$.

For these measurements, two detectors, STAR and PHENIX, will be simultaneously used, and their functions are complementary as shown in Appendix II. Expected event rates given in this paper are for the detector STAR.

Let us consider the hadronic reaction, $\mathrm{pp} \rightarrow$ (hadron or gauge boson) $+\mathrm{X}$. When both initial protons are longitudinally polarized, we measure an observable ALL defined as:

$$
A_{L L}=\left(1 / P^{2}\right)\left(N^{++}-N^{+-}\right) /\left(N^{++}+N^{+-}\right) .
$$

If one QCD subprocess is dominant:

$$
A_{L L} \sim P_{a} \cdot P_{b} \cdot \hat{a}_{L L}(a+b \rightarrow c+d),
$$

where $\hat{\mathrm{a}}_{\mathrm{LL}}$ in various reactions are shown in Appendix III.5 


\section{1}

Measurements with Barrel EMC and Shower Maximum Detector

The proposed barrel EM calorimeter is a lead-scintillator sampling calorimeter. It is located inside the aluminum coil of the STAR solenoid and covers $|\eta| \leq 1.0$ and $2 \pi$ in azimuth, thus matching the acceptance for full TPC tracking. At $\eta \sim 0$, the amount of material in front of the EMC is $\sim 0.5$ radiation lengths $\left(X_{0}\right)$. The inner radius is 2.20 meters, and the overall length is 6.20 meters.

A detector with fine spatial resolution will be placed at a depth of approximately 5 $\mathrm{X}_{0}$, near the location of the maximum number of shower electrons for photons of 3-5 GeV, to allow for the detection of direct photons. It will reject background photons emanating from decaying $\pi^{\circ}$ mesons having $\mathrm{p}_{\mathrm{T}} \leq 20 \mathrm{GeV} / \mathrm{c}$ by examining the transverse shower profile at this depth. The radial space allotted for this device is $25 \mathrm{~mm}$.

(Reference - EMC CDR2)

\subsubsection{Jet Production at $200 \mathrm{GeV}$}

Several QCD subprocesses contribute to the cross section for jet production:

a) gluon-gluon scattering at low $\mathrm{p}_{\mathrm{T}}$,

b) gluon-quark scattering at medium $\mathrm{p}_{\mathrm{T}}$ (above $\sim 20 \mathrm{GeV} / \mathrm{c}$, and

c) quark-quark elastic scattering at $\mathrm{p}_{\mathrm{T}}$.

At low $\mathrm{p}_{\mathrm{T}}$ :

$$
A_{L L}=\left[\Delta G\left(x_{1}\right) / G\left(x_{1}\right)\right] \times\left[\Delta G\left(x_{2}\right) / G\left(x_{2}\right)\right] \times \hat{a}_{L L}(g g \rightarrow g g) .
$$

The $\hat{\mathrm{a}}_{\mathrm{LL}}$ is expected to be large, $\hat{\mathrm{a}}_{\mathrm{LL}}=0.8$ at $90^{\circ}$.

\subsubsection{Di-Jet Production at $200 \mathrm{GeV}$}

The advantage over the single jet is the kinematic constraint on the momentum fractions, $x_{i}$, of the two partons.

\begin{tabular}{|c|c|c|}
\hline \multicolumn{3}{|c|}{ Jet + Jet Events } \\
\hline $\mathrm{p}_{\mathrm{T}}$ & $|\eta|$ & $\mathrm{N}_{\text {pair }}$ \\
\hline$\geq 10$ & $\leq 0.3$ & $1 \cdot 10^{8}$ \\
\hline$\geq 20$ & $\leq 0.3$ & $3 \cdot 10^{6}$ \\
\hline
\end{tabular}

\subsubsection{Direct- $\gamma$ Production at $200 \mathrm{GeV}$}

Direct photons are produced through the $q \bar{q}$ annihilation subprocess and the quark-gluon Compton subprocess, (qg $\rightarrow \gamma q$ ). The Compton process is the dominant one in pp interactions. Then,

$$
A_{L L}=\left[\Delta u\left(x_{1}\right) / u\left(x_{1}\right)\right] \cdot\left[\Delta G\left(x_{2}\right) / G\left(x_{2}\right)\right] \cdot \hat{a}_{L L}(q g \rightarrow \gamma q),
$$

where $\Delta u(x) / u(x)=\left(u_{+}(x)-u_{-}(x)\right] /\left[u+(x)+u_{-}(x)\right] \Delta u(x)$ being the helicity distribution of the quark, $\Delta G(x) / G(x)=\left[G_{+}(x)-G_{-}(x)\right] /\left[G_{+}(x)+G_{-}(x)\right]$, where $\Delta G(x)$ is the helicity distribution carried by gluon fields. 
For example, $\Delta u(x) / u(x) \approx 0.4$ at $x_{q}=0.2$ (from EMC - SMC), $\hat{a}_{L L}=0.6$ at $90^{\circ}$ scattering. Then we have $A_{L L}=0.2 \times \Delta G / G$, and $\delta(\Delta G / G)=5 \times \delta A_{L L}$.

The estimated $\delta A_{L L}$ at $\sqrt{\mathrm{s}}=200 \mathrm{GeV}$ for $\mathrm{p}_{\mathrm{T}}=10$ to $20 \mathrm{GeV}, \Delta \mathrm{y} \pm 1$,

\subsubsection{Direct- $y+$ Jet}

$$
\begin{gathered}
\delta A_{L L} \sim \pm 0.006 \\
\delta(\Delta G / G) \sim \pm 0.03
\end{gathered}
$$

For the $\mathrm{p}_{\mathrm{T}}$ acceptance of 10 to $20 \mathrm{GeV}, \mathrm{x}_{1}$ and $\mathrm{x}_{2}$ vary from 0.1 to 0.2 at $\sqrt{\mathrm{s}}=200$ $\mathrm{GeV}$. The expected number of events is 9,000 corresponding to

$$
\delta A_{L L} \sim \pm 0.03
$$

\subsubsection{W土and $Z^{\circ}$ Production at $500 \mathrm{GeV}$}

a. Parity-Violating Asymmetry

The observable $A_{L}(P V)$ is defined as, $A_{L}=\left(N^{-}-N^{+}\right) /\left(N^{-}+N^{+}\right)$, where $-(t)$ are minus (plus) helicity. For $\mathrm{W}^{+}$,

$$
A_{L}=\frac{\Delta u\left(x_{1}\right) \bar{d}\left(x_{2}\right)-(u \leftrightarrow \bar{d})}{u\left(x_{1}\right) \bar{d}\left(x_{2}\right)+\bar{d}\left(x_{1}\right) u(x 2)} .
$$

When the helicities of both beams are the same, we define another observable as:

$$
\mathrm{A}_{\mathrm{LL}}^{\mathrm{PV}}(\mathrm{y})=\frac{\left[\Delta \mathrm{u}\left(\mathrm{x}_{1}\right) \overline{\mathrm{d}}\left(\mathrm{x}_{2}\right)-\Delta \overline{\mathrm{d}}\left(\mathrm{x}_{2}\right) \mathrm{u}\left(\mathrm{x}_{1}\right)\right]-(\mathrm{u} \leftrightarrow \overline{\mathrm{d}})}{\left[\mathrm{u}\left(\mathrm{x}_{1}\right) \overline{\mathrm{d}}\left(\mathrm{x}_{2}\right)-\Delta \mathrm{u}\left(\mathrm{x}_{1}\right) \Delta \overline{\mathrm{d}}\left(\mathrm{x}_{2}\right)\right]+(\mathrm{u} \leftrightarrow \overline{\mathrm{d}})} .
$$

For $\mathrm{y}=0, \mathrm{~A}_{\mathrm{L}}^{\mathrm{W}^{+}}=1 / 2(\Delta \mathrm{u} / \mathrm{u}-\Delta \overline{\mathrm{d}} / \mathrm{d})$ and $A_{L}^{W^{-}}=1 / 2(\Delta d / d-\Delta \bar{u} / \mathrm{u})$. The results of predictions $^{3}$ for $\mathrm{W}^{ \pm}$production are shown in Fig. 3 with polarized sea quarks ${ }^{4}$ and $\Delta \overline{\mathrm{u}}=\Delta \overline{\mathrm{d}}$. In the case of W-production, one observes a drastic difference between the cases $\Delta \overline{\mathrm{u}} \neq 0$ and $\Delta \overline{\mathrm{u}}=0$.

\section{Figure 3}

The parity violating asymmetry $\mathrm{A}_{\mathrm{LL}}^{\mathrm{PV}}$ vs. $\mathrm{y}$ for $\mathrm{W}^{+}$and $\mathrm{W}^{-}$production at $\sqrt{\mathrm{s}}=500 \mathrm{GeV}$. Solid lines correspond to non-zero sea quark polariza tions where as dashed lines correspond to $\Delta \overline{\mathrm{u}}=\Delta \overline{\mathrm{d}}=0$.

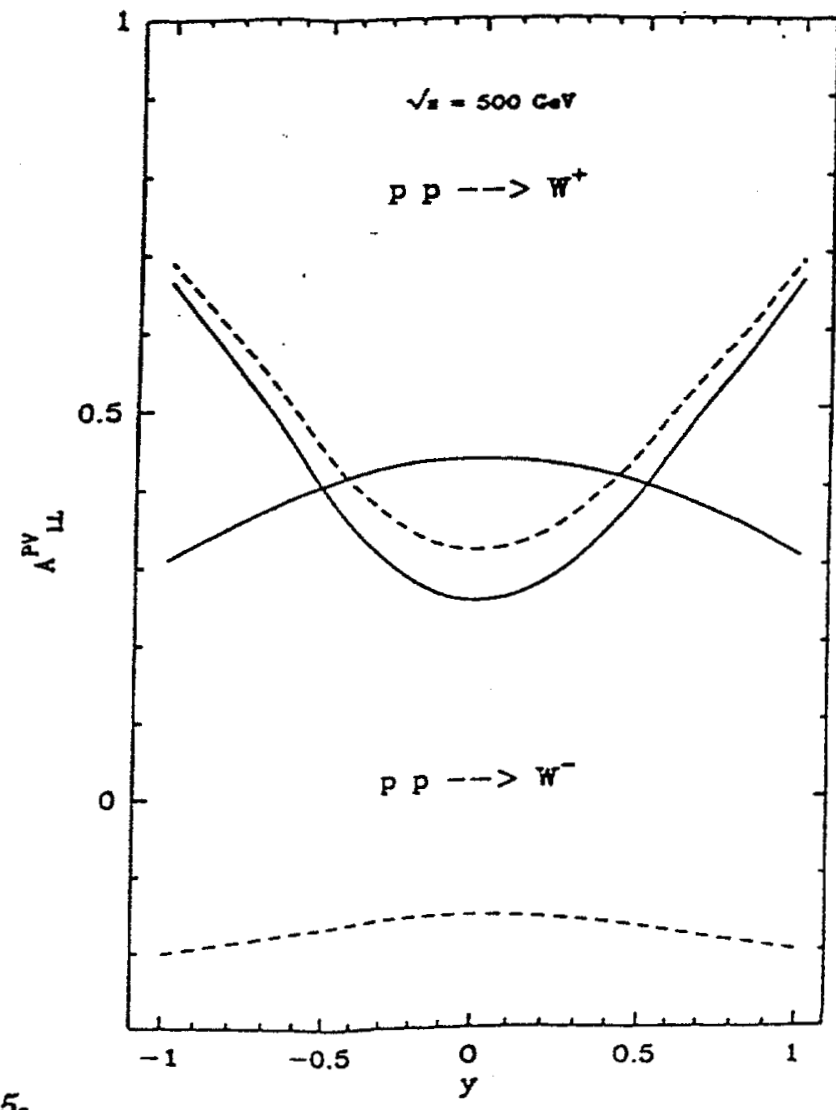


b. Parity-Conserving Asymmetry

$$
\text { For } \mathrm{W}^{+}, A_{\mathrm{LL}}-\frac{\Delta \mathrm{u}\left(\mathrm{x}_{1}\right) \Delta \overline{\mathrm{d}}\left(\mathrm{x}_{2}\right)}{\mathrm{u}\left(\mathrm{x}_{1}\right) \overline{\mathrm{d}}\left(\mathrm{x}_{2}\right)}
$$

A similar expression is for $\mathrm{W}$ - production by permuting $\mathrm{u}$ and $\mathrm{d}$.

\subsubsection{Measurements of $h_{1}(x)$ in $Z^{\circ}$ Production}

(Quark Tranversity Distribution in Polarized Proton)

A complete quark-parton model of the nucleon requires three quark distributions and quark spin density matrix is given as:

$$
P(x)=1 / 2\left[f_{1}(x)+g_{1}(x) \vec{s}_{/ /} \cdot \vec{\sigma}+h_{1}(x) \vec{s}_{\perp} \cdot \bar{\sigma}\right],
$$

where $f_{1}(x)$ is related to the longitudinal momentum distribution of quarks in the nucleon, $g_{1}(x)$ is related to the helicity distribution in a polarized nucleon, and $h_{1}(x)$ is related to the correlation between left-handed and right-handed quarks.

$h_{1}(x)$ can be determined by measuring the transverse spin correlation $A_{T T}\left(A_{N N}\right)$ in $Z^{\circ}$ production.

In terms of $h_{1}(x), A_{T T}\left(A_{N N}\right)$ is given as:

$$
A_{T T}\left(A_{N N}\right)=\hat{a}_{\mathrm{TT}} \frac{\sum_{i}\left(a_{i}^{2}-b_{i}^{2}\right) h_{1 i}\left(x_{1}\right) \bar{h}_{1 i}\left(x_{2}\right)}{\sum_{i}\left(a_{i}^{2}+b_{i}^{2}\right) f_{1 i}\left(x_{1}\right) \bar{f}_{1 i}\left(x_{2}\right)},
$$

where $a_{i}$ and $b_{i}$ are the vector and axial couplings of the $Z^{\circ}$ to the quark of flavor $i, \hat{a}_{\text {TT }}$ is the partonic double-spin asymmetry, $\hat{\mathrm{a}}_{\mathrm{TT}}=1$ at the vicinity of $\theta_{\text {c.m. }}=\pi / 2$ and $\phi_{\text {c.m. }}=0$.

The statistical error will be $\Delta A_{T T}=\left(1 / P^{2}\right) \cdot 0.025$. At a first approximation $h_{1}=$ $\mathrm{g}_{1}$, then $\left|\mathrm{A}_{\mathrm{TT}} / \hat{\mathrm{a}}_{\mathrm{TT}}\right|-\left|\mathrm{A}_{\mathrm{LL}}\right|$.

\subsection{Measurements with Barrel, One Endcap, and Shower Maximum Detector}

An endcap calorimeter is to be placed inside the iron pole pieces. The endcap increases solid angle and acceptance and allows better measurements of the following reactions.

\section{Detecting the Direct- $y$ and the "Away-Side" Jet}

In order to measure $\Delta G(x)$, the gluon spin structure function, both the direct- $\gamma$ and the "away-side" jet must be detected in coincidence so that the kinematics of the incoming partons can be calculated.

The Compton and annihilation subprocesses both involve $2 \rightarrow 2$ scatterings. The incoming partons are assumed to have fractions $x_{1}$ and $x_{2}$ of the beam momentum and collide colinearly. Then $\mathrm{x}_{1}$ and $\mathrm{x}_{2}$ are given in terms of pseudorapidity as:

$$
\mathrm{x}_{1} \simeq\left(2 \mathrm{p}_{\mathrm{T}} / \sqrt{\mathrm{s}}\right)\left(\mathrm{e}^{\eta_{1}}+\mathrm{e}^{\eta_{2}}\right) / 2, \mathrm{x}_{2} \simeq\left(2 \mathrm{p}_{\mathrm{T}} / \sqrt{\mathrm{s}}\right)\left(\mathrm{e}^{-\eta_{1}}+\mathrm{e}^{-\eta_{2}}\right) / 2 \text {. }
$$


Figure 4 shows the $\mathrm{x}$ coverage for $\mathrm{XT}=0.1$ as functions of the direct- $\gamma$ and jet pseudorapidities.

W's and Z Production at $500 \mathrm{GeV}$

$\mathrm{W}^{+} \quad 74,000$ events

$W^{-} \quad 22,000$ events

For $\mathrm{W}$ 's the detected electron (or positron) has $\mathrm{p}_{\mathrm{T}}>20 \mathrm{GeV} / \mathrm{c}$

Z $\quad 5,300$ events

For $\mathrm{Z}$ at least one of the detected electrons has $\mathrm{p}_{\mathrm{T}}>20 \mathrm{GeV} / \mathrm{c}$

In particular, many $Z$ events are needed for a reasonable measurement of $h_{1}(x)$.
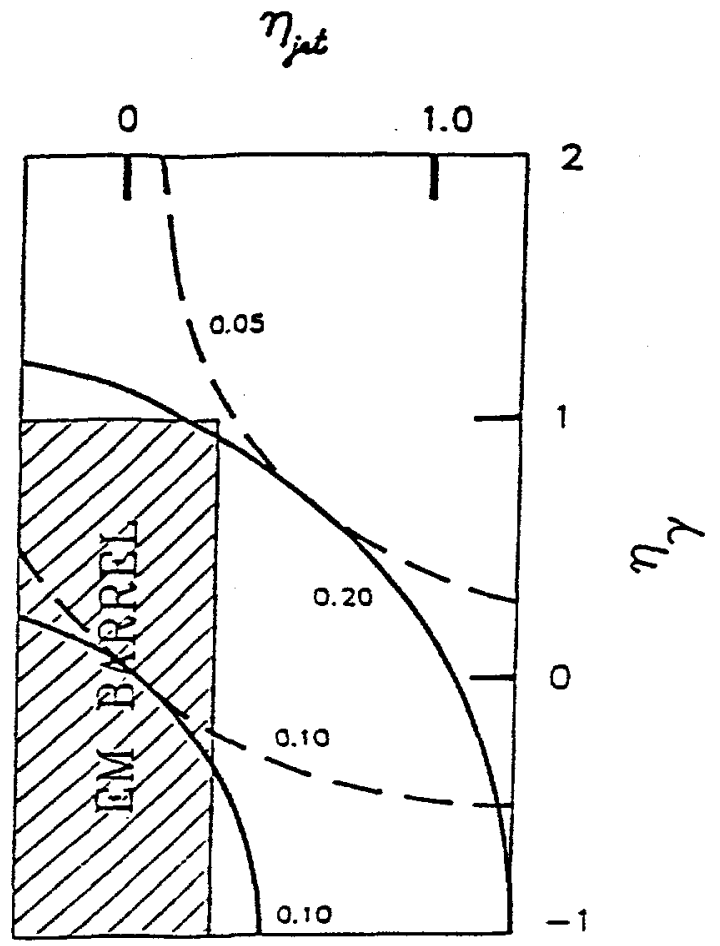

Figure 4 Plots of $x_{1}$ and $x_{2}$ as a function of the direct- $\gamma$ and jet pseudorapidities. The solid lines represent contours for $\mathrm{x}_{1}$ and the dashed lines are contours for $\mathrm{x}_{2}$.

* Work supported by the U.S. Department of Energy, Division of High Energy Physics Contract W-31-109-ENG-38.

\section{References}

1) H. Huang et al., Phys. Rev. Lett. to appear.

2) The Electromagnetic Calorimeter for the Solenoidal Tracker at RHIC, PUB-5380 (1993).

3) C. Bourrely and J. Soffer, Phys. Lett. B314, 132 (1993).

4) A. D. Martin, W. J. Sterling, and R. G. Roberts, Preprint RAL-92-021 (1992).

5) C. Bourrely, J. Ph. Guillet, and J. Soffer, Nucl. Phys. B361, 72 (1991);

C. Bourrely, J. Soffer, F. M. Renard, and P. Taxil, Phys. Rep. 177, 319 (1989). 


\section{APPENDIXI}

The integrated luminosities used are:

$$
\mathrm{Ldt}=8 \cdot 10^{38} \mathrm{~cm}^{-2} \text { at } 500 \mathrm{GeV}=800 \mathrm{pb}^{-1} \text {, }
$$

and

which means

$$
\mathrm{Ldt}=3.2 \cdot 10^{38} \mathrm{~cm}^{-2} \text { at } 200 \mathrm{GeV}=320 \mathrm{pb}^{-1} \text {, }
$$

$$
100 \text { days of running (4 } 11^{6} \text { sec. } 50 \% \text { efficiency). }
$$

\section{APPENDIX II \\ SPIN Physics -- PHENIX/STAR Experiments}

Reaction

PHENIX

STAR

(EMC:Barrel only)

\begin{tabular}{ccc}
\hline $\mathrm{W}^{ \pm} \rightarrow \mathrm{e}^{ \pm}+\mathrm{X}$ & $12,000 \mathrm{~W}^{+}, 4,000 \mathrm{~W}$ & $62,400 \mathrm{~W}^{+}, 13,700 \mathrm{~W}$ \\
\hline $\mathrm{Z}^{\circ} \rightarrow \mathrm{e}^{+} \mathrm{e}^{-}$ & $\begin{array}{c}\text { Very small acceptance } \\
160 \mathrm{Z}^{\circ}\end{array}$ & $\begin{array}{c}\text { Transversity } \mathrm{h} 1(\mathrm{x}), \\
\text { sea-quark helicity }(\mathrm{x}) \\
3100 \mathrm{Z}^{\circ}\end{array}$ \\
\hline Direct $\gamma(\Delta \mathrm{G})$ & $\begin{array}{c}\text { High Resolution } \\
\text { Resolve } \pi^{\circ} \mathrm{pT} \leq 25 \mathrm{GeV} / \mathrm{c}\end{array}$ & $\begin{array}{c}\text { Barrel and SMD } \\
\text { pT } \leq 25 \mathrm{GeV} / \mathrm{c} \\
\text { Large solid angle }\end{array}$ \\
\hline Jet & Leading Particles & $|\eta| \leq 0.5$ \\
\hline Di-Jets & Leading $-\pi^{\circ}$ Pairs & $\geq 10^{6}$ di-jets \\
\hline Direct- $\gamma+$ Jet & & $\Delta \mathrm{G}(\mathrm{x}), \mathrm{x} \leq 0.2$ \\
\hline $\mathrm{J} / \psi$ & $1200 \chi_{2}$ Events & Sizable rates for $\mathrm{e}^{+} \mathrm{e}^{-}$ \\
$\chi_{2} \rightarrow \mathrm{J} / \psi+\gamma$ & &
\end{tabular}




\section{APPENDIXIII}

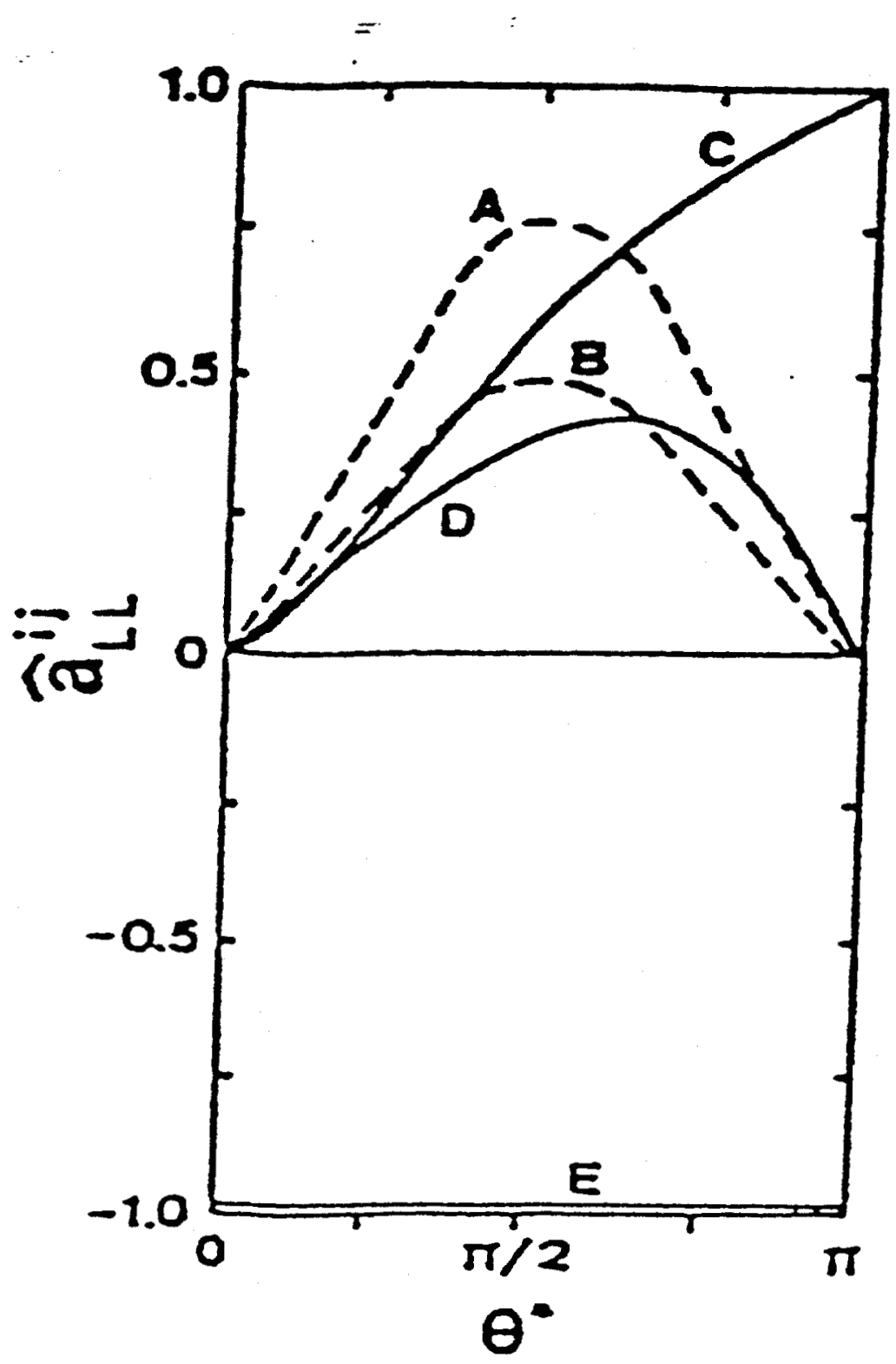

\section{A $[99-99$ \\ $B\left[\begin{array}{l}d d-d d \\ u v-u v\end{array}\right.$}

$c\left[\begin{array}{l}u d-u d \\ u \bar{d}-4 \bar{d} \\ d \bar{u}-d \bar{u} \\ 9 g-9 g \\ 9 g-98\end{array}\right.$

$D\left[\begin{array}{l}u \bar{u}-u \bar{u} \\ d \bar{d}-d \bar{d}\end{array}\right.$

$E\left[\begin{array}{l}9 g-q \bar{q} \\ q \bar{q}-9 g \\ u \bar{u}-d \bar{d} \\ d \bar{d}-4 \bar{u} \\ q \bar{q}-g r\end{array}\right.$

\section{DISCLAIMER}

This report was prepared as an account of work sponsored by an agency of the United States Government. Neither the United States Government nor any agency thereof, nor any of their employees, makes any warranty, express or implied, or assumes any legal liability or responsibility for the accuracy, completeness, or usefulness of any information, apparatus, product, or process disclosed, or represents that its use would not infringe privately owned rights. Reference herein to any specific commercial product, process, or service by trade name, trademark, manufacturer, or otherwise does not necessarily constitute or imply its endorsement, recommendation, or favoring by the United States Government or any agency thereof. The views and opinions of authors expressed herein do not necessarily state or reflect those of the United States Government or any agency thereof. 